\title{
Factors Influencing Value of Children and Intergenerational Relations in Times of Social Change: Analyses From Psychological and Socio-Cultural Perspectives: Introduction to the Special Issue
}

\author{
Gisela Trommsdorff* \\ University of Konstanz, Germany \\ Uichol Kim \\ Chung-Ang University, Republic of Korea \\ Bernhard Nauck \\ Chemnitz University of Technology, Germany
}

Research on the reasons for and the implications of the recent dramatic sociodemographic changes in most parts of the world has increased in several disciplines such as sociology, anthropology, and demographics. In psychology, however, this topic has only recently been rediscovered and is presently gaining increasing importance in the area of applied psychology. Recently a special section on this topic was published by the American Psychological Association

* Address for correspondence: Gisela Trommsdorff, Faculty of Sciences, Department of Psychology, Developmental Psychology and Cross Cultural Psychology, University of Konstanz, Box D 14, 78457 Konstanz, Germany. Email: gisela.trommsdorff@uni konstanz.de

This special issue has been prepared based on a grant from the German Research Council (Deutsche Forschungsgemeinschaft, DFG) to Gisela Trommsdorff, University of Konstanz, and Bernhard Nauck, Technical University of Chemnitz, Germany (principal investigators) for the study "Value of Children and Intergenerational Relations" (TR 169/9 1,2,3: NA 164/9 $1,2,3)$. Theoretical and applied issues underlying ongoing social change in the international context are major concerns of applied psychology. Applied Psychology: An International Review is in a unique position to deal with some of the related issues. We are grateful to Gilad Paz, the Managing Editor of $A P / I R$, who helped to coordinate the various tasks required by a special issue. We also wish to express our appreciation to the reviewers. Furthermore, we would like to thank Holly Bunje for her valuable comments on each of the included papers and Rozalia Horvath for copyediting the manuscripts. 
dealing with demography and fertility (e.g. David \& Russo, 2003), pointing out several still under-researched issues in the area of applied psychology. However, this topic is not new. More than 30 years ago, economists and demographers started a large international study on the "value of children" (Arnold, Bulatao, Buripakdi, Chung, Fawcett, Iritani, Lee, \& Wu, 1975; Fawcett, 1977) that means reasons why parents want (or do not want) children (e.g. economic, social, or other reasons). This original "value of children" (VOC) study was motivated by an interest in understanding why high fertility rates occur in several regions of the world and lead to massive increases in the population size. This study first dealt with the question of to what degree economic factors explain fertility; later on, the interest shifted to include additional theoretically relevant variables such as family structure and psychological factors such as beliefs. This VOC study asked the question why young couples want to have children in order to specify facets of both the positive and negative value of children. The underlying hypothesis was that an economic and socio-normative value of children in contrast to an emotional value of children is related to high fertility, thus introducing psychological variables into a previously economic approach.

After more than three decades, the topic of value of children has been taken up again in order to carry out a large cross-cultural study. Unlike the previous research, this work was not limited to the question of fertility but also included important questions pertaining to childbearing and intergenerational relations (Nauck, 2005; Trommsdorff, Zheng, \& Tardif, 2002). This revised approach to preconditions and consequences of the value of children is based on large samples of persons from three biologically related generations (grandmothers, mothers, adolescent children) (300 families in each country) plus a sample of young mothers with a preschool child (300 in each country). So far, the countries included in this study are Germany, France, Turkey, Israel, Indonesia, Republic of Korea, People's Republic of China, India, and South Africa. Some of these countries have already participated in the original VOC study (Turkey, Indonesia, Republic of Korea, and Germany) and therefore provide data sets which now can be studied with respect to socioeconomic and cultural change.

The papers included in this special issue deal with selected aspects of the presently ongoing study on the value of children with each paper focusing on one country. The first two papers take into account the issue of social change while the subsequent papers deal with issues of the psychological structure of the value of children, and intergenerational relations from the perspective of the transmission of values and the exchange of intergenerational support.

Cigdem Kagitcibasi who has been a member of the team from the original VOC study and her co-author Bilge Ataca take advantage of the fact that Turkey has participated in both waves of data collection; they compare 
results from both data sets with regard to changes in the value of children for Turkish mothers. They contextualise their findings in a general model of family change and prove its fruitfulness in the case of Turkey which is a country of massive social change and extreme social disparities.

Uichol Kim, Young-Shin Park, Young-Eun Kwon, and Jaisun Koo present data from the ongoing VOC study and relate these to the data from the original study carried out in the Republic of Korea. In spite of the social, economic, and political changes that have occurred in the past 30 years, the emphasis on the maintenance of strong relational bonds persists. The psychological benefits are the most important reasons for having a child. Personal and financial constraints are the most important reasons for not having a child. These results challenge the economic and utilitarian models and suggest the importance of understanding the psychological, relational, and cultural factors.

The paper by David Lackland Sam, Karl Peltzer, and Boris Mayer focuses on the values of children and preferences regarding family size in South Africa where fertility and also infant mortality are especially high, many young mothers are not married, and economic problems abound. The authors can demonstrate a specific factor structure for the African sample and different preferences regarding family size across generations. Generation-specific relations among the value of children and preferences regarding family size point to the relevance of the value of children for fertility.

Colette Sabatier and Lyda Lannegrand-Willems address the question whether relations between the value of children, family values, and intergenerational relations exist in a French sample. Their data indicate most proximity between mothers and adolescents. Grandmothers influence mothers (their adult daughters) through values and parental goals (obedience and autonomy), thus taking an active part in the intergenerational transmission of values.

The paper by Beate Schwarz, Gisela Trommsdorff, Isabelle Albert, and Boris Mayer deals with the question of intergenerational relations over the life span on the basis of theoretical assumptions on intergenerational solidarity for a German sample. The analyses reveal positive relations between family values, relationship quality, and support to parents. Perceived reciprocity was associated with the exchange of intergenerational support, and imbalance in support.

In summary, this special issue brings together studies from different countries on selected aspects of the value of children and intergenerational relations including theoretical and applied aspects of ongoing processes of sociodemographic change in the international context. The next step will be to carry out systematic cross-cultural comparisons among these various samples in order to test theoretical models regarding the value of children and intergenerational relations. 


\section{REFERENCES}

Arnold, F., Bulatao, R.A., Buripakdi, C., Chung, B.J., Fawcett, J.T., Iritani, T., Lee, S.J., \& Wu, T. S. (1975). The value of children: A cross national study. Vol. 1. Intro duction and comparative analysis. Honolulu, HI: East West Population Institute.

David, H.P., \& Russo, N.F. (2003). Psychology, population, and reproductive behavior. American Psychologist, 58, 193196.

Fawcett, J.T. (1977). The value and cost of children: Converging theory and research. In L.T. Ruzicka (Ed.), The economic and social supports for high fertility (Vol. 2, pp. 91 114). Canberra, Australia: Department of Demography.

Nauck, B. (2005). Changing value of children: An action theory of fertility behavior and intergenerational relationships in cross cultural comparison. In W. Friedlmeier, P. Chakkarath, \& B. Schwarz (Eds.), Culture and human devel opment: The importance of cross cultural research to the social sciences (pp. 183 202). Hove, UK: Psychology Press.

Trommsdorff, G., Zheng, G., \& Tardif, T. (2002). Value of children and intergen erational relations in cultural context. In P. Boski, F.J.R. van de Vijver, \& A.M. Chodynicka (Eds.), New directions in cross cultural psychology. Selected papers from the Fifteenth International Conference of the International Association for Cross Cultural Psychology (pp. 581 601). Warszawa: Polish Psychological Association. 